\title{
Nano-scale 3-D characterization of materials using FIB-SEM
}

\author{
K. Dovidenko, D. Ellis, J. Grande, R. A. Potyrailo \\ General Electric Global Research Center, Niskayuna NY 12309
}

Nano-scale characterization in three dimensions can provide essential information regarding structural, optical or chemical behavior of materials. Dual-column Focused Ion Beam-Scanning Electron Microscopes (FIB-SEMs) have been successfully used for microstructural characterization of materials and devices through sequential milling and imaging ('slice-and-view' technique). Combined with image reconstruction, the approach demonstrates ability to study key microstructural features in 3-D [1-2].

In this paper, we describe the challenges and achievements of the 'slice-and-view' approach applied to complex material systems and taken to nano-scale. We will demonstrate results of technique application to various materials such as metal-polymer, nano-scale gamma-prime phase in superalloys, nanotubes and nanorods. One of the examples is metal nano-particles imbedded into polymer matrix on a glass substrate. The typical dimensions of the particles are $50-75 \mathrm{~nm}$. The sliceand-view sequences have been collected to study spatial distribution of the Au particles in 3-D. Typical slices were about $25 \mathrm{~nm}$ thick. The study was carried out using an FEI Nova 200 Nano Lab FIB-SEM. Amira 3.1 software package has been used for 3-D reconstruction.

Figure 1 shows 3 -D reconstruction of the $3.4 \times 3.4 \times 0.75 \mu \mathrm{m}$ data set with the sample being crosssectioned normal to $\mathrm{Z}$ direction in the image. The data obtained from such 3-D characterization is critical for correct nano-particles density evaluation. Typically, particle concentration is estimated from SE images of the FIB cut surface (Figure 2). However, we need to take into account that SE images are 2-D representations of 3-D objects, since metal particles exhibit contrast even if located 15-20 nm below the polymer surface. The image analysis algorithm does take this into account, but only the 3-D data from FIB-SEM 'slice-and-view' sequence provides complete information on spatial distribution and particles concentration.

\section{References}

[1] M. D. Uchic, M. Croeber, R. Wheeler IV, F. Scheltens, D. M. Dimiduk, Microsc. Microanal. 10 (Suppl. 2), 2004

[2] R.E.A. Williams, M. D. Uchic, D. M. Dimiduk, H.L. Fraser, Microsc. Microanal. 11 (Suppl. 2), 2005 


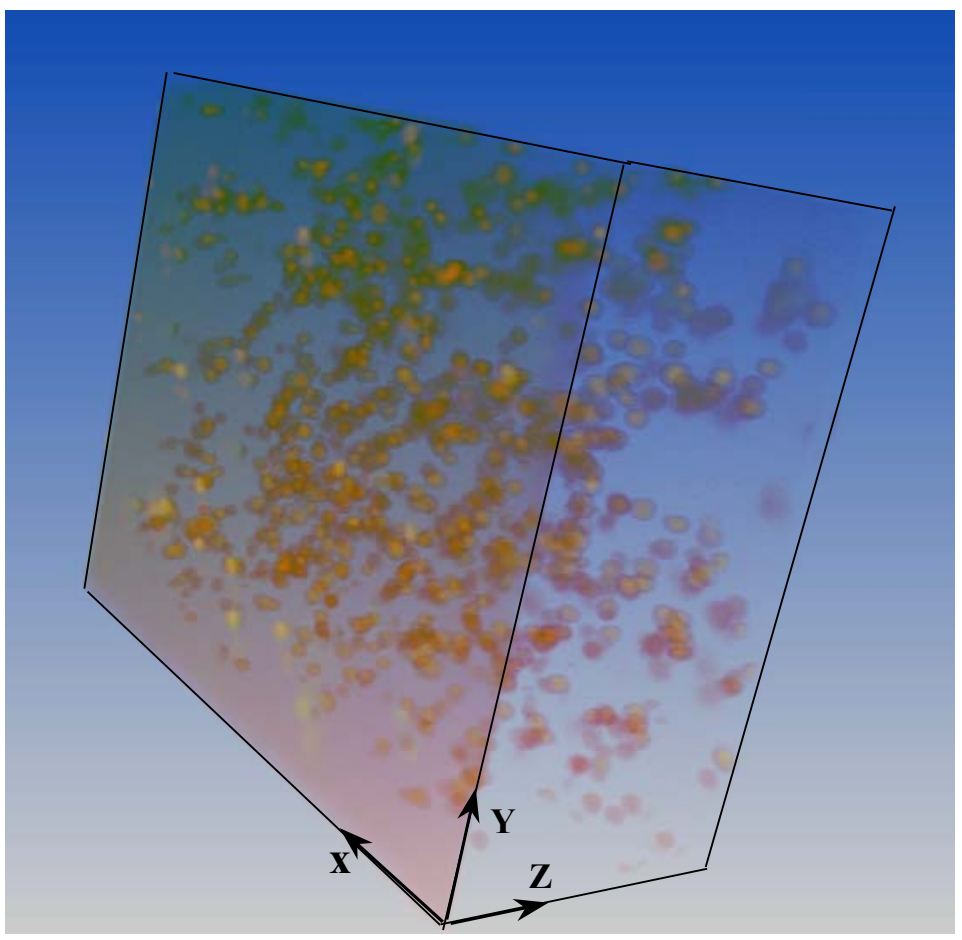

FIG. 1. 3-D representation of data set from FIB serial sectioning normal to $\mathrm{Z}$ direction in the picture. Lateral dimensions of the cut: $\mathrm{X}=\mathrm{Y}=3.4 \mu \mathrm{m}$.

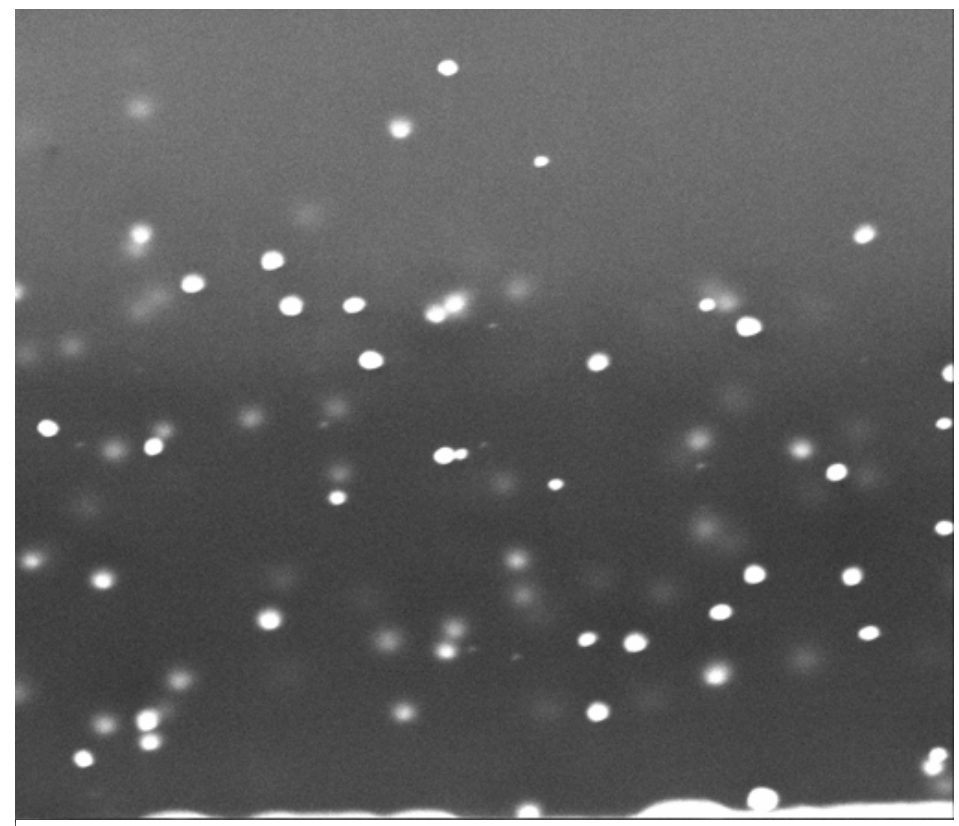

FIG. 2. SE image of the cross-section made by FIB showing $\sim 75 \mathrm{~nm}$ Au particles in PVA. Same magnification as in the $3-\mathrm{D}$ rendering (Figure 1). 